This report was prepared as an account of work sponsored by an agency of the United States Government. Neither the United States Government nor any agency thereof, nor any of their employees, makes any warranty. express or implied, or assumes any legal hability or responsibility for the accuracy, completeness, or usefulness of any information, apparatus, product, or process diselosed, or represents that its use would not infringe privately owned rights. Reference herein to any specific commercial product, process, or service by trade name, trademark, manufacturer, or otherwise does not necessarily constitute or imply its endorsement, recommendation, or favoring by the United States Government of any agency thereof. The views and opinions of authors expressed herein do not necessarily state or reflect those of the United States Government or any agency thereof.

\title{
MEASUREMENTS OF 1/f NOISE IN A-Si:H PIN DIODES AND THIN-FILM-TRANSISTORS.
}

\author{
Gyuseong Cho, J.S. Drewery, I. Fujieda, T. Jing, \\ S.N. Kaplan, V. Perez-Mendez, S. Qureshi, D. Wildermutl, \\ Physics Division \\ Lawrence Berkeley Laboratory \\ University of California, Berkeley, CA 94720
}

and R.A. Street

Xerox PARC, Palo Alto, CA 94304

May 1990

This work was supported by the Director, Office of Energy Research, Office of High Energy and Nuclear Physics, Division of High Energy Physics, and Office of Health and Environmental Research, Division of Physics and Technological Research of the U.S. Department of Energy under Contract No. DE-AC03-76SF00098. 


\section{MEASUREMENTS OE $1 / f$ NOISE IN A-Si:H PIN DIODES AND}

THIN-FILM-TRANSISTORS.

GYUSEONG CHO^, J.S. DREWERY*, I. FUJIEDA*, T. JING*, S.N. KAPLAN*

V. PEREZ-MENDEZ*, S. QURESHI*, D. WIIDERMUTH*, and R.A. STREET ${ }^{\star} \star$

* Lawrence Berkeley Iaboratory, Berkeley, CA 94720

** Xerox PARC, Palo Alto, CA 94304

\section{ABSTRACT}

We measured the equivalent noise charge of $\mathrm{a}-\mathrm{Si}: \mathrm{H}$ pin diodes $(5-45$ $\mu \mathrm{m}$ i-layer) with a pulse shaping time of $2.5 \mu \mathrm{sec}$ under reverse biases up to $30 \mathrm{~V} / \mu \mathrm{m}$ and analyzed it as a four component noise source. The frequency spectra of $1 / f$ noise in the soft-breakdown region and of the Nyquist noise from contact resistance of diodes were measured. Using the conversion equations for a $C R-R C$ shaper, we identified the contact resistance noise and the 1/f noise as the main noise sources in the low bias and high bias regions respectively. The $1 / f$ noise of a-Si:H TFTs with channel length of $15 \mu \mathrm{m}$ was measured to be the dominant component up to $-100 \mathrm{kHz}$ for both saturation and linear regions.

\section{INTRODUCTION}

In previous papers[1-5], we and other groups have covered various applications of reversed biased pin a-Si:H diodes to charged particles, xray and $\gamma$-ray detection. For charged particle detection in particular, we showed that pin diodes with thin $\mathrm{p}$ and $\mathrm{n}$ blocking layers and thick $(5-45$ $\mu \mathrm{m})$ i regions could serve as efficient position sensitive detectors. Since the collected signal for minimum ionizing particles is $\sim 80$ electron-hole pairs/ $\mu \mathrm{m}$ of the $i$-layer [1], it is obviously important to design the diodes and associated TFT amplifiers with a minimum amount of electronic noise generation. For thin a-Si diodes, 1/f type noise measurements and theory have been published. [6 8] In this paper we discuss measurements of various noise sources originating from thick reverse biased pin diodes and from the thin-film-transistors(TFTs) that we propose to use for readout of pixel or strip detector configurations.

\section{NOISE AND REVERSE CURRENT IN A-Si:H PIN DIODES}

\section{II (a) Experimental}

Sample a-Si:H pin diodes were fabricated by the standard PECVD method at Xerox PARC (Palo Alto, CA) and at Glasstech Solar Inc. (Wheatridge, Co) . They consist of five deposited layers or glass substrates: the bottom layer is a metallic electrode fonmed by $\mathrm{Cr}$ or $\mathrm{tin}$ oxide; the next three layers are $n^{*}$-type $(\sim 50 \mathrm{~nm})$, intrinsic $(5 \sim 45 \mu \mathrm{m})$, ard $\mathrm{p}^{*}$-type ( 50 nm) a-Si:H layers made by PECVD. The last layer is a top electrode made by evaporation of $\mathrm{Cr}$, $\mathrm{Pd}$, or $\mathrm{Al}$, and it is etched to form circular contacts of radius between $1.5 \sim 3 \mathrm{~mm}$. Sample diodes have equivalent capacitances $10 \sim 250 \mathrm{pF}$ determined by the area of the top electrode and the i-layer thickness.

Since the diodes are reverse biased winen used as radiation detectors, the dark current and noise charactoristics are investigated under this condition. Diode noise sas measured by the two configurations shown in Fig. 1. The first method Fig. 1-(a) measures the equivalent input noise charge in the time domain. The measurement time is set by a pulse shaping amplifier, usually a CR-RC circuit, which performs a differentiation and integration for shaping the signal pulse. The second method Fig. 1-(b) is a 
set-up to determine the frequency spectrum of the output voltage noise $\left.\left\langle v^{2}\right\rangle\right\rangle$ following a charge sensitive amplifier. Noise data were recorded on a digital oscilloscope and their Eourier transforms were calculated in a PC. Aliasing due to the digital sampling is removed by a Butterworth filter for each measurernent frequency range set by the scope. The output voltage noise is related to the input equivalent current noise by the equation,

$$
\left\langle v_{0}^{i}\right\rangle=\frac{A_{w}^{2}}{\left(\omega C_{i n}\right)^{2}} \times\left\langle i_{i n}^{2}\right\rangle=\frac{K^{2}}{(2 \pi f)^{2}} \times\left\langle i_{1 n}^{2}\right\rangle
$$

where $\omega$ is $2 \pi f, C_{i r}$ is the dynamic input capacitance of the preamplifier, $A_{w}$ is the voltage gain of the wide band amplifier and $K$ is the charge-tovoltage conversion gain of the measurement system $\left(\sim 3 \times 10^{12} \mathrm{~V} / \mathrm{Coul}\right)$.

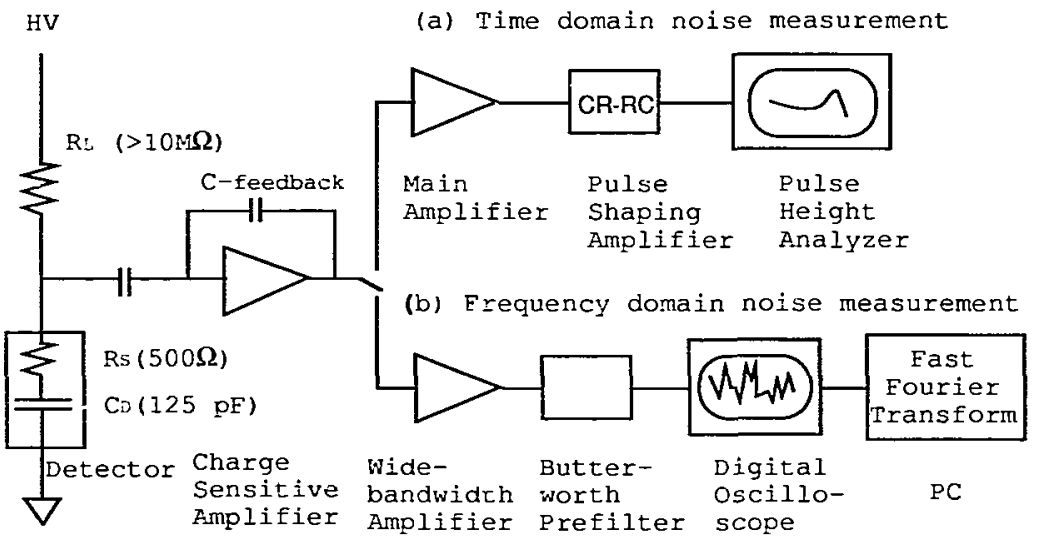

Fig. 1. Sciematic of noise measurement set-up

\section{II (b) Results and Discussion}

When a-Si:H pin diodes are usec as raciation detectors, full cepletion is required in order to ensure full charge collection. Usually the operating bias level is set below the point where the pre-breakdown starts shown by a rapid increase in noise. Most of the diodes we have tested show sirilar I-V and noise characteristics; therefore the discussion here will be confined to measurements on a $26 \mu \mathrm{m}$ thick pin diode having 3 man top electrode radius and an equivalent capacitance of $125 \mathrm{pF}$.

Fig. 2 shcws the reverse current density $(o)$ and the noise charge $(\cdot)$ measured with a $2.5 \mathrm{Hsec}$ shaping time. The measured noise is flat up to a bias of $15 \mathrm{~V} / \mu \mathrm{m}$ and then starts to increase repidly. The increasing noise has deen attributed to some reversible soft-breakdown[9] at the high electric field m-p-i junction area. Our present measurements show that the noise frequency spectra in this bias region are $1 / f$ and are proportional to the reverse current density. This result is similar to an earlier report on I/f noise from a-Si:H Schottky diodes. [7]

The measured noise data (*) fits very well to the calculated total noise (solid line) From 0 to $30 \mathrm{~V} / \mu \mathrm{m}$ bias by the equation

$$
\mathrm{N}_{\text {tot }}^{2}=\mathrm{N}_{\leq Y \mathrm{~s}}^{2}+\mathrm{N}_{\mathrm{B}_{\mathrm{s}}}^{2}+\mathrm{N}_{\text {sict }}^{2}+\mathrm{N}_{1 / \pm}^{2}
$$

there the terms in the right hand sice are separately measured or calcula- 
ted from their spectra by equations (3), (5) and (7) . [10]

(a) system noise $\mathrm{N}^{2}$ sys : It originates mainly from the charge sensitive preamplifier(Amtek A225) and is measured to be 1300 electrons rms by connecting an $125 \mathrm{pF}$ capacitor in place of the diode, in parallel with a ioad resistor $10 \mathrm{M} \mathbf{\Omega}$, whose contribution to the total noise is negligible.

(b) contact resistance noise $\mathrm{N}^{2} \mathrm{Ps}_{\mathrm{s}}$ : The diode has a finite contact resistance $R_{s}$ of $\sim 500 \Omega$ in series with its equivalent capacitance as illustrated in Fig. 1, which contributes 1500 electrons rms to the system noise. We confirmed that $R_{\mathbf{s}}$ is originated from the evaporated Pd top contact. Simulation by a $500 \Omega$ resistor in series with an 125 pF capacitor gives the same noise charge as the unbiased sample diode and also agrees well with the estimated noise value by the following equation for a simple CR-RC shaping amplifier, [10]

$$
N_{R_{s}}^{2}=\left(\frac{e}{q}\right)^{2} \times \int_{0}^{\infty} \frac{\left\langle v_{R_{s}}^{2}\right.}{\Delta f} C_{D}^{2}|G|^{2} d f=\left(\frac{e}{q}\right)^{2} \times 4 k T R_{s} \times \frac{C_{D}^{2}}{8 \tau}
$$

where $e$ is $2.718, q$ is the electronic charge, $\tau$ is the shaping time 12.5 $\mu s e c),\left\langle v_{R s}^{2}\right\rangle$ is the noise voltage source due to the resistor $R_{s}$, and $|G|$ is the transfer function of CR-RC shaper. [10]
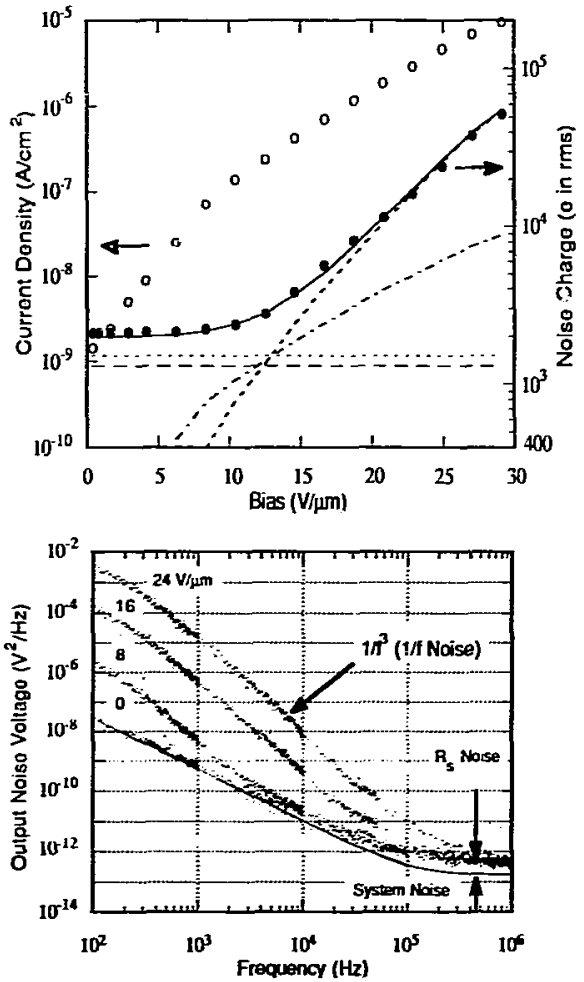

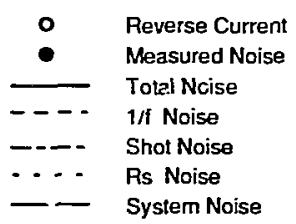

Fig. 2. Reverse current and Noise measurement of a reverse biased 26 $\mu \mathrm{m}$ thick pin diode. Measured noise data $(\cdot)$ is fitted well by the sum (solid line) of four noise components; (a) system noise, (b) contact resistance noise, (c) shot noise of diode, and (d) $1 / f$ noise of diode.

Eig. 3. Noise spectra of a reverse biased $26 \mu \mathrm{th}$ thick pin diode. $1 / \mathrm{f}^{3}$ dependency in output noise voltage spectra is equivalent to $1 / E$ when converted into a parallel current noise sorrce in the diocle by the equation (1). Nyquist noise from the contact resistance equivalent to 500 $\boldsymbol{\Omega}$ is shown as a mite spectrum by an arrow in the figure. 


$$
\mid G^{2}=\frac{(\omega \tau)^{2}}{\left(1+(\omega \tau)^{2}\right)^{2}}
$$

Fig. 3 shows the excess Nyquist noise component at high frequency due to this series resistance $R_{s}$.

(c) Shot noise $N_{\text {sinot }}^{2}$ Shot noise of the diode in the time domain is calculated from the measured reverse current $I_{r}$ by the equation,

$$
\mathbf{N}_{\text {shor }}^{2}=\left(\frac{e}{q}\right)^{2} \times \int_{0}^{\infty} \frac{\left\langle i_{\text {shar }}^{2}\right\rangle}{\Delta f} \frac{\mid \mathrm{G}^{2}}{\omega^{2}} d f=\left(\frac{e}{q}\right)^{2} \times 2 q I_{r} \times \frac{\tau}{8}
$$

(d) $1 / f$ noise $\mathbb{N}_{1 / \mathrm{f}}^{2}$ : The measured output noise voltage spectra $\left\langle v^{2}\right\rangle$ under various bias conditions are plotted in Fig. 3 in which $1 / \mathbb{f}^{3}$ is found to be dominant at low frequency. Equation (1) show that $1 / f^{3}$ dependence of output noise voltage is equivalent to a $1 / f$ spectrum of input current noise $\left\langle i{ }^{2}{ }_{\text {in }}\right\rangle$. The measured magnitude of the $1 / \mathrm{f}^{\mathfrak{3}}$ noise power is proportional to the square of the reveise current so the equivalent input current noise of the $1 / \pm$ component can be empirically represented by

$$
\left\langle i_{i n}^{2}\right\rangle=K_{d} \times \frac{I_{r}^{2}}{f} \Delta E
$$

where $K_{0}$ is a constant $\left(\sim 3.6 \times 10^{-9}\right)$ and the measured $k_{0}$ value decreases from $8 \times 10^{-7}$ to $8 \times 10^{-8}$ almost linearly as the inverse of the square of the i-layer thickness from $10 \mu \mathrm{m}$ to $45 \mu \mathrm{m}$. $K_{\mathrm{c}}$ is equivalent to $\mathrm{A} / \mathrm{N}$ in Hooge's empirical relation for $1 / f$ noise where $A$ is Hooge's constant $[11]$ - nd $N$ is the total number of charge carriers in the device. The $1 / f$ noise in the time domain is calculated from

$$
\mathrm{N}_{1 / \mathrm{E}}^{2}=\left(\frac{\mathrm{e}}{\mathrm{q}}\right)^{2} \times \int_{0}^{\infty} \frac{\left\langle\mathrm{i}_{1 / \mathrm{s}}^{2}\right\rangle}{\Delta \mathrm{f}} \frac{\mid \mathrm{G}^{2}}{\omega^{2}} \mathrm{df}=\left(\frac{\mathrm{e}}{\mathrm{q}}\right)^{2} \times \mathrm{K}_{\mathrm{d}} \mathrm{I}_{\mathrm{s}}^{2} \times \sigma \tau^{2}
$$

where the lower frequency limit of the integration was assumed to be about $100 \mathrm{~Hz}$ which is equivalent to the trapping level at $\sim 0.6 \mathrm{eV}$ below conduction band. The above equation shows that the $1 / \mathrm{f}$ noise cuntribution can be reduced by decreasing the reverse current, or by reducing the surface field, or by using a smaller shaping time $\tau$.

Since the shot noise is linearly proportional to the current and $1 / f$ noise is proportional to the square of the reverse current, the shot noise is dominant at the lower bias and $1 / \mathrm{f}$ noise is dominant at higher bias. At higher bias the diodes start to breakdown irreversibly and are permanently damaged. Mist of diodes with i-layer thickness range of $5-45 \mu \mathrm{m}$ show similar characteristics to the one discussed here. However some poorer quality diodes have lower onset bias for sharp breakdown behavior.

\section{1/E NOISE TN A-Si:F: THTN-FILM-TRANSISTORS}

\section{III (a) Experimental}

Sample a-Si:H TFTs were fabricated at Xerox PARC. They are of the staggered inverted type which has a structure shown in Fig. 4. The gate insulator is $S_{1} \mathrm{~N}_{\mathrm{s}}$ of thickness $0.3 \mu \mathrm{m}$ and the channel length is $15 \mu \mathrm{m}$. The channel width ranges from $16 \mu \mathrm{m}$ to $256 \mu \mathrm{m}$.

$I-V$ characteristics and noise spectra were measured in a shielded probe station. A HP 4145 semiconductor parameter analyzer was used to measure the dC I-V curves and transfer characteristics. A HP 3561A spectrum analyzer with a low noise current amplifier was used to measure the spectra 
of the noise current at each bias condition. Then the equivalent input voltage noise $\left\langle v^{2}\right.$ in $\rangle$ was calculated by the following relation.

$$
\left\langle v_{i n}^{2}\right\rangle=\frac{\left\langle i_{d}^{2}\right\rangle}{g_{m}^{2}}
$$

where $\left\langle i^{2}\right\rangle$ is the measured drain current fluctuation and $g_{m}$ is the transconductance at the bias point.

\section{III (b) Results and discussion}

The dc drain current and its fluctuation change linearly with the channel width of the sample TFTs so the discussion here is given for a 'IFT with channel width of $128 \mu \mathrm{m}$. Fig. 5 shows I-V curves for the sample IFT. The measured field effect mobility in the saturation region has a range of $0.4 \sim 0.6 \mathrm{~cm}^{2} / \mathrm{Vsec}$, which corresponds to a range of band mobility of $13 \sim$ $18 \mathrm{~cm}^{2} /$ Vsec as calculated from the Xerox TFT model $[12,13]$ used in the PSPICE program.

1/f type noise is found to be dominant in the bias and frequency range of interest. The equivalent input voltage noise in the saturation
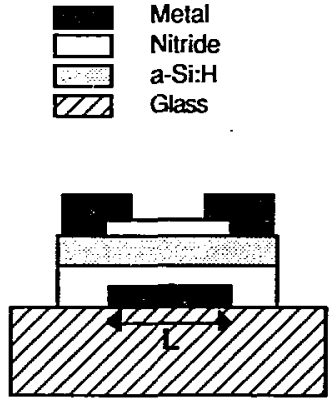

Eig. 4. Scherratic diagram of a-Si:H thin-iilm-transistors made at Xerox

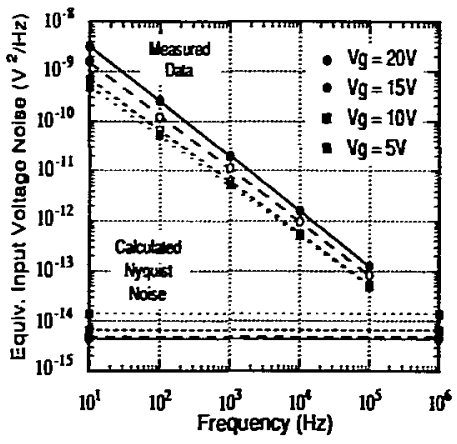

Fig. 6. Gate bias dependence of noise spectre of a-Si:H TFT of $L=15 \mu \mathrm{m}$ and $w=128$ un when $v_{\mathrm{d}}=20 \mathrm{v}$

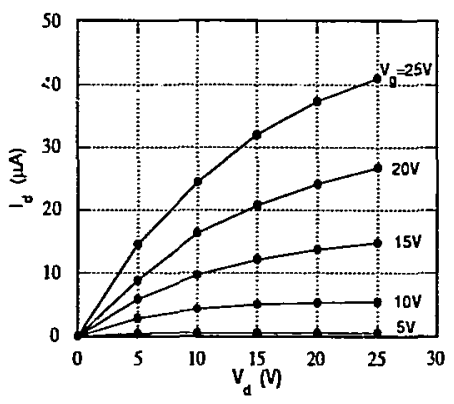

Fig. 5. I-V curves of a-Si:H TFT with $\mathrm{L}=15 \mu \mathrm{m}$ and $\mathrm{w}=128 \mu \mathrm{m}$

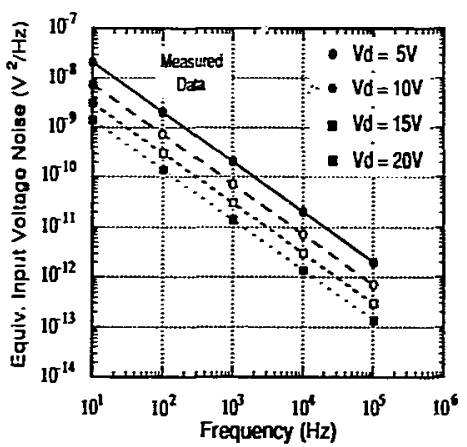

Eig. 7. Drain bias dependence of noise spectra of $a-S i=H$ TFT of $L=15 \mu \mathrm{m}$ and $\mathrm{W}=128 \mu \mathrm{m}$ when $\mathrm{v}_{\mathrm{g}}=15 \mathrm{~V}$ 
region as shown in Fig. 6 is generally expressed[14] by the equation

$$
\left\langle v_{i n}^{2}\right\rangle=K_{t} \times \frac{I_{d}^{\beta}}{f^{\alpha}} \Delta f
$$

where $I_{d}$ is dc cirain-source current. Measured $\alpha$ and $K_{c}$ increase slightly from 0.97 to 1.1 and from $1 \times 10^{-6}$ to $3 \times 10^{-6}$ respectively as the gate bias increases from $5 \mathrm{~V}$ to $20 \mathrm{~V}$. Measured $\beta$ changes from 0.41 to 0.85 when the frevuency changes from $1 \mathrm{~Hz}$ to $-100 \mathrm{kHz}$. The range of $\boldsymbol{\beta}$ agrees with the empirical Si-JFET model. [14] The Nyquist (thermal) noise due to the finite channel resistance of the IFT was calculated by the following equation in the saturation region,

$$
\left\langle v_{\text {Nyquist }}^{2}\right\rangle=\frac{4 k T}{(3 / 2) g_{m}} \Delta f
$$

Fig. 7 shows a steady decrease of the equivalent input voltage noise as drain bias increases from $5 \mathrm{~V}$ to $20 \mathrm{~V}$ when $\mathrm{V}_{\mathrm{G}}=15 \mathrm{~V}$. Similar effects have been observed in crystal-Si MOSFET. [-5]

\section{IJ CONCLUSION}

In reverse biased pin diodes, series contact resistance noise is dominant at lcw bias and $1 / \mathrm{f}$ type noise is dominant in the soft-breakdown region. In order to minimize noise, reduction of contact resistance as well as reverse current is important. For a-Si:H TFTs, 1/f type noise is found to be dominant over the whole operating frequency range. Whether the $1 / f$ type noise is an interface effect or a bulk effect in both a - Si:H pin diode and TFTs is still unclear and requires further investigation.

\section{ACKNOWLEDGEMENTS}

We would like to thank to $S$. Nelson at Xerox PARC for making sample TFTs and $\mathrm{J}$. Xi and R. E. Hollingsworth at GSI for making sample a-Si:H pin diodes. Also we specially thank to $M$. Hack at Xerox PARC for valuable ciscussion on TFTs. This work was supported by the Director, Office of High Energy and Nuclear Physics, and Ofice of Health and Environmental Research of the U.S. Department of Energy under Contract No. LE-AC03-76SF00098.

\section{REFERENCES}

[1] V. Perez-Mendez, et al., Nucl. Irstr, and Neth., A250, 195 (1987)

[2] V. Perez-Hendez, et al., Nucl. Instr. and Heth., A273, 127 (1988)

[3] V. Perez-Mendez, et al., MRS. Symp. Proc. 149, 621 (1989)

[4] B. Equer and A. Kaxar, Nucl. Instr. and Meth., A271, 574 (1988)

[5] T. Pochet, et al., MRS. Symp. Proc. 149, 661 (1989)

[6] E.Z. Bathaei and J.C. Anderson, Phil. Hag., B 55, 87 (1987)

[7] F.Z. Bathaei, et al., Phil. Mag., B 57, 259 (1988)

[8] A.D'Anico, et al., I. Non-Cryst. Sol., $77 \& 78,499$ (1985)

[9] R.A. Gibson, et al.s Appl. Phys., 21, 307 (1980)

[10] F.S. Goulding and D.A. Iandis, IFEE Trans. Nuc. Sci., 29, 1125 (1982)

[11] E.N. Eooge, Rep. Prog. Phys. 44, 479 (1981)

[12] M. Hacs;, et al., IEFE Trans. Elect. Dev., 36, 2764 (1989)

[13] M. Shur, et ai.., J. Appl. Phys., 66, 3371 (1989)

[14] P.R. Gray and R.G. Meyer, Analysis and Desion of Analog ICs

John wiley and Sons, (2nd Ed.), pp.647, New York (1984)

[15] N. Wutoh, et al., Solid State Electronics, 31, 1675 (1988) 